\title{
Optimization of Resonances in Photonic Crystal Slabs
}

\author{
Robert P. Lipton ${ }^{a}$, Stephen P. Shipman ${ }^{a}$, and Stephanos Venakides ${ }^{b}$ \\ ${ }^{a}$ Louisiana State University, Baton Rouge, LA, USA \\ ${ }^{b}$ Duke University, Durham, NC, USA
}

\begin{abstract}
Variational methods are applied to the design of a two-dimensional lossless photonic crystal slab to optimize resonant scattering phenomena. The method is based on varying properties of the transmission coefficient that are connected to resonant behavior. Numerical studies are based on boundary-integral methods for crystals consisting of multiple scatterers. We present an example in which we modify a photonic crystal consisting of an array of dielectric rods in air so that a weak transmission anomaly is transformed into a sharp resonance.
\end{abstract}

Keywords: photonic crystal, resonance, optimization, transmission

\section{OVERVIEW}

Resonant electromagnetic scattering behavior in photonic crystal slabs (as sheets, films, or walls) is closely connected to anomalous behavior of the transmission of energy through them. It has been observed empirically and through numerical simulations that leaky waveguide modes, Fabry-Perot bandgap resonances, and other resonant phenomena coincide in the frequency domain with narrow regions of enhanced transmission or reflection of incident waves (see Refs. 1, 2, 3, and 4).

In this report, we develop a method for designing infinite periodic photonic crystal slabs that have certain desired electromagnetic resonant properties. Motivated by the connection between resonant behavior and transmission, our method focuses on modifying or optimizing those properties of a photonic crystal slab that have a relation to its transmission coefficient. We do this by developing the variational calculus of the transmitted energy as a function of the material parameters. We restrict our analysis to lossless materials in a two-dimensional reduction, in which the design parameters are the dielectric permittivity $\epsilon$, the magnetic permeability $\mu$, and the structure of the crystal.

Our numerical methods are based on free Green's functions and boundary integrals and are therefore suited to crystals whose period consists of a finite number of disjoint scatterers with smooth boundaries. In particular, we apply the variational calculus to the manipulation of resonant properties of crystals that are built of circular dielectric rods embedded in a contrasting dielectric medium.

Cox and Dobson (Refs. 5 and 6) have devised a gradient-search method for optimizing and creating bandgaps for two-dimensional photonic crystals that are infinite in both directions. This is based on the generalized gradient of the bandwidth derived from the variational calculus of the dispersion relation of the periodic structure. Resonant scattering behavior in photonic crystal slabs is known to be related to complex dispersion relations (Ref. 4): source fields at real frequencies near a complex eigenvalue produce resonant scattering in the crystal and enhanced or inhibited transmission. A method based on the manipulation of these dispersion relations holds promise for engineering crystal slabs with desired resonant properties. In this study, we approach the problem by manipulating the transmission as a function of the structure and material properties of dielectric crystals.

Further author information: (Send correspondence to S.P.S)

R.P.L.: E-mail: lipton@math.lsu.edu, Telephone: 1225578 1569; supported by grants AFOSR F49620-02-1-0041 and NSF DMS-0296064

S.P.S.: E-mail: shipman@math.lsu.edu, Telephone: 1225578 1674; supported by LA Board of Regents grant LEQSF(200306)-RD-A-14

S.V.: E-mail: ven@math.duke.edu, Telephone 1919660 2815; supported by grants ARO-DAAD19-99-1-0132 and NSF DMS-0207262. 


\section{THE MATHEMATICAL FRAMEWORK}

Our photonic crystal slab consists of an array of lossless rods perpendicular to the $x y$-plane embedded in a lossless medium of contrasting dielectric permittivity $\epsilon$ and magnetic permeability $\mu$. The array is periodic in the $y$ direction and truncated to a finite width in the $x$ direction. In a cross-section (Fig. 1), one period of the structure consists of a finite number of domains $D_{j}, j=1, \ldots, n$, with boundaries $\partial D_{j}$, and we put $D=\cup_{j=1}^{n} D_{j}$. We assume that each rod, as well as the exterior medium is homogeneous. We consider free time-harmonic electromagnetic fields that are polarized so that either the electric field points out of the plane and the magnetic field is transverse to the rods (the transverse magnetic, or TM, case) or the magnetic field points out of the plane (the TE case). In either case, we denote the out-of-plane component by $\psi(x, y) e^{-i \omega t}$, where $\omega$ is the reduced frequency*, and the Maxwell equations reduce to the Helmholtz equation

$$
\nabla^{2} \psi+\epsilon \mu \omega^{2} \psi=0
$$

This equation holds in the interiors of the regions $D_{j}$ and in the exterior medium, where the values of $\epsilon$ and $\mu$ take on different constant values. In the TM case, the Maxwell equations imply the following matching conditions for the interior and exterior limiting values of the electric field component $\psi$ on $\partial D$.

$$
\begin{aligned}
\psi_{\text {int }} & =\psi_{\text {ext }} \\
\mu_{\text {ext }} \partial_{n} \psi_{\text {int }} & =\mu_{\text {int }} \partial_{n} \psi_{\text {ext }}
\end{aligned}
$$

In the TE case, $\mu_{\text {ext }}$ and $\mu_{\text {int }}$ are replaced by $\epsilon_{\text {ext }}$ and $\epsilon_{\text {int }}$, so it is sufficient to work out the analysis for the TM case.

We assume further that the field $\psi$ is pseudoperiodic in $y$, that is

$$
\psi(x, y)=\tilde{\psi}(x, y) e^{i \beta y}
$$

in which $\tilde{\psi}(x, y)$ has period $2 \pi$ in the variable $y$. Such fields are called Bloch waves. The pseudo-periodicity allows us to restrict the analysis to one period of the structure, which is the strip $\mathcal{S}=\{(x, y):-\infty<x<\infty, 0 \leq y \leq 2 \pi\}$ (Fig. 1).

A pseudo-periodic radiating fundamental solution of the Helmholtz equation in a homogeneous medium $(\epsilon$ and $\mu$ constant) is given by $G(x-\hat{x}, y-\hat{y})$, where $G(x, y)$ satisfies

$$
\nabla^{2} G+\epsilon \mu \omega^{2} G=-\sum_{k=-\infty}^{\infty} \delta(x, y-2 \pi k) e^{2 \pi k i \beta}
$$

For values of $\beta$ and $\omega$ such that $\epsilon \mu \omega^{2}-(m+\beta)^{2} \neq 0$ for all integers $m$, its Fourier form is

$$
G(x, y)=-\frac{1}{4 \pi} \sum_{m=-\infty}^{\infty} \frac{1}{\nu_{m}} \exp \left(\nu_{m}|x|+i(m+\beta) y\right)
$$

The Green's function is built of a finite number of propagating plane-wave modes, for which $\nu_{m}=i \kappa_{m}$, $\kappa_{m}>0, m=m_{1}, \ldots, m_{2}$, and infinitely many decaying (evanescent) modes, for which $\nu_{m}<0$. The Green's function, together with Green's identities reduce much of the theory of the Helmholtz equation to boundary integrals and shows us that the asymptotic behavior of bounded solutions in the strip $\mathcal{S}$ as $|x| \rightarrow \infty$ is simply a superposition of plane waves traveling to the right and left:

$$
\psi \sim \sum_{m=m_{1}}^{m_{2}}\left(a_{m}^{ \pm} e^{i \kappa_{m} x}+b_{m}^{ \pm} e^{-i \kappa_{m} x}\right) e^{i(m+\beta) y} \quad(x \rightarrow \pm \infty)
$$

\footnotetext{
${ }^{*}$ We define the reduced frequency by $\omega=f L / c$, where $c$ is the speed of light, $L$ is the period of the structure, and $f$ is the frequency in cycles per time. The reduced spatial variables $(x, y)$ are related to the physical variables $(X, Y)$ by $(x, y)=2 \pi(X, Y) / L$.
} 


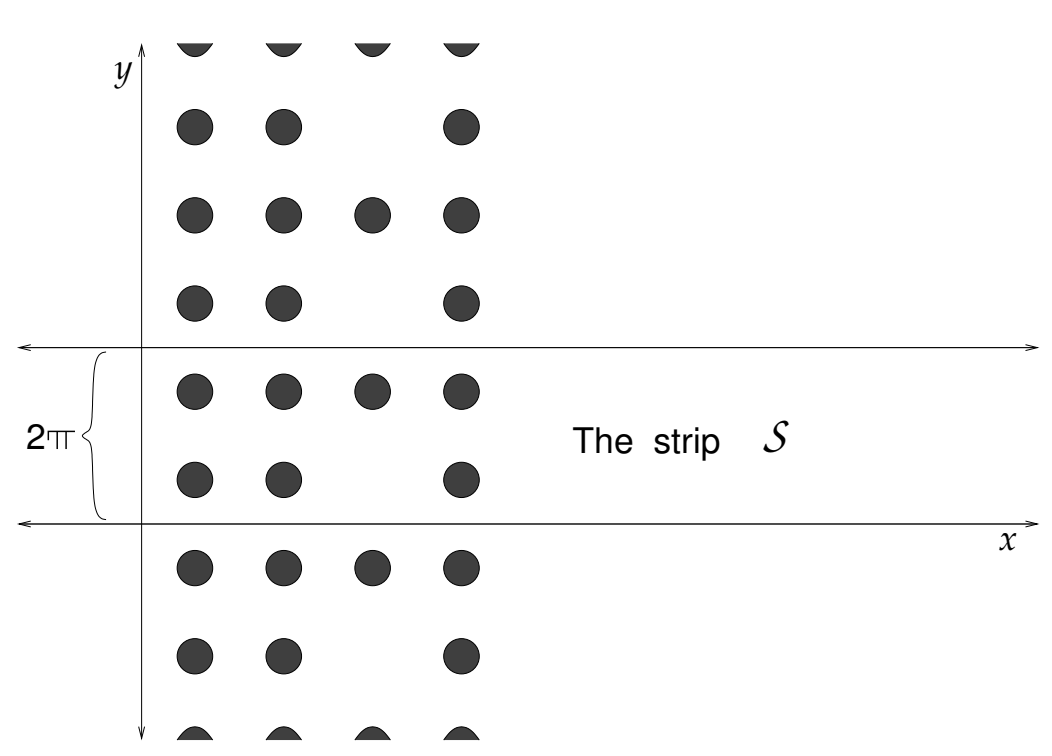

Figure 1. A cross-section of a photonic crystal slab consisting of an array of homogeneous dielectric rods standing perpendicular to the $x y$-plane. The rod structure is periodic in the $y$-direction, with period $2 \pi$, and extends indefinitely as $y \rightarrow \pm \infty$. The rod structure is finite in the $x$-direction. Exterior to the rods is a homogeneous material of contrasting dielectric permittivity extending to infinity to the right and left. Pseudo-periodic fields in the plane can be analyzed in the strip $\mathcal{S}=\{(x, y):-\infty<x<\infty, 0 \leq y \leq 2 \pi\}$ consisting of a single period of the dielectric permittivity function.

The solution approaches the asymptotic form exponentially as $|x| \rightarrow \infty$.

In the scattering problem that we study in this paper, a source field incident upon the slab at an angle $\theta$ from the left is given:

$$
\psi_{\text {inc }}=e^{i \kappa_{\bar{m}} x} e^{i(\bar{m}+\beta) y},
$$

where $\bar{m}$ is an integer such that $(\bar{m}+\beta)^{2}<\epsilon \mu \omega^{2}$. This is a plane wave whose angle of incidence with the slab is $\theta=\arcsin \frac{\bar{m}+\beta}{\sqrt{\epsilon \mu} \omega}$. $\beta$ can always taken to lie in the interval $[-1 / 2,1 / 2)$, and $\bar{m}=0$ and $\beta=0$ corresponds to normal incidence.

The total field $\psi$ has the asymptotic form

$$
\begin{aligned}
& \psi \sim e^{i \kappa_{\bar{m}} x} e^{i(\bar{m}+\beta) y}+\sum_{m=m_{1}}^{m_{2}} r_{m} e^{-i \kappa_{m} x} e^{i(m+\beta) y} \quad(x \rightarrow-\infty) \\
& \psi \sim \sum_{m=m_{1}}^{m_{2}} t_{m} e^{i \kappa_{m} x} e^{i(m+\beta) y} \quad(x \rightarrow \infty)
\end{aligned}
$$

in which $r_{m}$ and $t_{m}$ are the coefficients of the modes of the fields reflected by the slab and transmitted through it, respectively.

\section{THE VARIATIONAL CALCULUS}

Let $x_{0}$ be any positive number large enough so that $D$ is contained in the truncated $\operatorname{strip} \Omega=\left\{(x, y):-x_{0}<\right.$ $\left.x<x_{0}, 0 \leq y \leq 2 \pi\right\}$; let $\Gamma_{-}$and $\Gamma_{+}$denote the left and right boundaries of $\Omega: \Gamma_{ \pm}=\left\{\left( \pm x_{0}, y\right): 0 \leq y \leq 2 \pi\right\}$; put $\Gamma=\Gamma_{-} \cup \Gamma_{+}$; and let $n$ denote the outward-pointing normal vector to $\Gamma$. 
Let $\psi$ be a pseudo-periodic solution of the Helmholtz equation with TM matching conditions:

$$
\left\{\begin{array}{l}
\nabla^{2} \psi+\epsilon \mu \omega^{2} \psi=0 \quad \text { away from } \partial D \\
\psi_{\text {int }}=\psi_{\text {ext }} \\
\mu_{\text {ext }} \partial_{n} \psi_{\text {int }}=\mu_{\text {int }} \partial_{n} \psi_{\text {ext }}
\end{array}\right\} \text { on } \partial D
$$

Let $\lambda$ be a smooth pseudo-periodic function in the plane: $\lambda=\tilde{\lambda} e^{i \beta y}$, with $\tilde{\lambda} 2 \pi$-periodic in $y$. Multiplying the Helmholtz equation in (1) by $\mu^{-1} \bar{\lambda}$, integrating by parts, and using the matching conditions, yields

$$
\int_{\Omega}\left(-\mu^{-1} \nabla \psi \cdot \nabla \bar{\lambda}+\epsilon \omega^{2} \psi \bar{\lambda}\right) d A+\int_{\Gamma} \mu^{-1} \bar{\lambda} \partial_{n} \psi \partial s=0
$$

The requirement that (2) hold for all $\lambda$ and truncated strips $\Omega$ is a weak formulation of the Helmholtz equation with the matching and boundary conditions given in (1) (see, for example, Ref. 7). It is equivalent to the system (1) for continuously twice-differentiable functions $\psi$ with well-defined limiting values on $\partial D$, that is, it implies the Helmholtz equation and automatically enforces the matching and pseudo-periodicity conditions.

We now state the problem of the scattering of a pseudo-periodic source field $\exp \left[i\left(\kappa_{\bar{m}} x+(\bar{m}+\beta) y\right)\right]$ incident upon the slab from the left:

$$
\left\{\begin{array}{l}
\int_{\Omega}\left(-\mu^{-1} \nabla \psi \cdot \nabla \bar{\lambda}+\epsilon \omega^{2} \psi \bar{\lambda}\right) d A+\int_{\Gamma} \mu^{-1} \bar{\lambda} \partial_{n} \psi d s=0 \\
\quad \text { for all pseudoperiodic } \lambda \text { and truncated strips } \Omega \\
\psi \sim e^{i \kappa_{\bar{m}} x} e^{i(\bar{m}+\beta) y}+\sum_{m=m_{1}}^{m_{2}} r_{m} e^{-i \kappa_{m} x} e^{i(m+\beta) y} \quad(x \rightarrow-\infty) \\
\psi \sim \sum_{m=m_{1}}^{m_{2}} t_{m} e^{i \kappa_{m} x} e^{i(m+\beta) y} \quad(x \rightarrow \infty)
\end{array}\right.
$$

The complex numbers $r_{m}$ and $t_{m}$ are the coefficients of the reflected and transmitted radiating plane waves.

The time-averaged energy flow of the transmitted field through $\Gamma_{+}$divided by the energy flow of the incident field is

$$
T=\frac{1}{2 \pi \kappa_{\bar{m}}} \Im \int_{\Gamma_{+}} \bar{\psi} \partial_{n} \psi d s
$$

which is independent of the location of $\Gamma_{+}$. We call this the transmission coefficient. The energy flow is computed as the real part of the complex time-harmonic Poynting vector.

We think of $\psi$ as depending on the material properties $\epsilon$ and $\mu$ and $T$ as a function of $\psi$ and consider the response of $T$ to variations in $\epsilon$ and $\mu$ for a fixed frequency and angle of incidence. Thus, the incident field $\exp \left[i\left(\kappa_{\bar{m}} x+(\bar{m}+\beta) y\right)\right]$ remains fixed but the reflected and transmitted fields will vary with the material parameters.

We derive the variational relations associated to this scattering problem by subtracting (3) and (4) from the equations obtained by inserting into (3) and (4) the perturbed quantities $\epsilon+\hat{\epsilon}, \mu^{-1}+\widehat{\mu^{-1}}, \psi+\hat{\psi}$ and $T+\hat{T}$ in place of $\epsilon, \mu^{-1}, \psi$, and $T$ :

$$
\left\{\begin{array}{c}
\int_{\Omega}\left[-\widehat{\mu^{-1}}(\nabla \psi+\nabla \hat{\psi}) \cdot \nabla \bar{\lambda}+\hat{\epsilon} \omega^{2}(\psi+\hat{\psi}) \bar{\lambda}\right] d A+ \\
\quad+\int_{\Omega}\left(-\mu^{-1} \nabla \hat{\psi} \cdot \nabla \bar{\lambda}+\epsilon \omega^{2} \hat{\psi} \bar{\lambda}\right) d A+\int_{\Gamma} \mu^{-1} \bar{\lambda} \partial_{n} \hat{\psi} d s=0 \\
\hat{\psi} \sim \sum_{m=m_{1}}^{m_{2}} \hat{r}_{m} e^{-i \kappa_{m} x} e^{i(m+\beta) y} \quad(x \rightarrow-\infty) \\
\hat{\psi} \sim \sum_{m=m_{1}}^{m_{2}} \hat{t}_{m} e^{i \kappa_{m} x} e^{i(m+\beta) y} \quad(x \rightarrow \infty),
\end{array}\right.
$$




$$
\hat{T}=\frac{1}{2 \pi \kappa_{\bar{m}}} \Im \int_{\Gamma_{+}}\left(\bar{\psi} \partial_{n} \hat{\psi}+\overline{\hat{\psi}} \partial_{n} \psi+\overline{\hat{\psi}} \partial_{n} \hat{\psi}\right) d s
$$

We have assumed that the support of $\widehat{\mu^{-1}}$ is inside $\Omega$ so that it does not contribute to the boundary integral along $\Gamma$.

We now seek a function $\lambda$ that represents the variation of the transmission functional. This means that $\lambda$ is chosen so that the part of (6) that is linear in $\hat{\psi}$ is equal to the imaginary component of the linear part of $(5)$ that involves $\hat{\psi}$ but not $\hat{\epsilon}$ or $\widehat{\mu^{-1}}$. Thus, we seek $\lambda$ such that, for all perturbations $\hat{\psi}$ satisfying the asymptotic conditions in (5),

$$
\Im\left[\int_{\Omega}\left(-\mu^{-1} \nabla \hat{\psi} \cdot \nabla \bar{\lambda}+\epsilon \omega^{2} \hat{\psi} \bar{\lambda}\right) d A+\int_{\Gamma} \mu^{-1} \bar{\lambda} \partial_{n} \hat{\psi} d s\right]=\mu_{\mathrm{ext}}^{-1} \Im \int_{\Gamma_{+}}\left(\bar{\psi} \partial_{n} \hat{\psi}+\overline{\hat{\psi}} \partial_{n} \psi\right) d s .
$$

(We have multiplied $\hat{T}$ by the constant $2 \pi \kappa_{\bar{m}} \mu_{\text {ext }}^{-1}$ for convenience.) Once we have found such a function $\lambda$, we use (5) and (6) to deduce that

$$
\hat{T}=\frac{\mu_{\text {ext }}}{2 \pi \kappa_{\bar{m}}} \Im \int_{\Omega}\left[\widehat{\mu^{-1}} \nabla \psi \cdot \nabla \bar{\lambda}-\hat{\epsilon} \omega^{2} \psi \bar{\lambda}\right] d A+\text { h.o.t. }
$$

where "h.o.t." refers to the higher order terms that are quadratic in the perturbation. This equation gives $\hat{T}$ in terms of $\hat{\epsilon}$ and $\widehat{\mu^{-1}}$. We remark that both sides of (7) are independent of the truncation boundary $\Gamma$, assuming that the perturbations $\hat{\epsilon}$ and $\hat{\mu}$ are contained in $\Omega$. That the left-hand side is independent of $\Gamma$ is evident from (5). That the right-hand side is independent of $\Gamma$ is seen as follows: $\hat{T}$ in equation (6) is (a constant multiple of) the difference of the energy flows of $\psi$ and $\psi+\hat{\psi}$ through $\Gamma_{+}$, which is independent of the $x$-value of $\Gamma_{+}$. The quadratic term $\Im \int_{\Gamma_{+}} \overline{\hat{\psi}} \partial_{n} \hat{\psi} d s$ is also independent of $\Gamma_{+}: \hat{\psi}$ is a radiating pseudo-periodic field; it has a Fourier expansion

$$
\hat{\psi}=\sum_{m=-\infty}^{\infty} a_{m} e^{\nu_{m} x} e^{i(m+\beta) y},
$$

where $\nu_{m}=i \kappa_{m}$ for the finite number of propagating modes and $\nu_{m}<0$ for the rest, and we compute

$$
\Im \int_{\Gamma_{+}} \overline{\hat{\psi}} \partial_{n} \hat{\psi} d s=\sum_{m=m_{1}}^{m_{2}} \kappa_{m}\left|a_{m}\right|^{2}
$$

which is independent of $x$.

We now show that a solution $\lambda$ to the following adjoint problem satisfies (7):

$$
\left\{\begin{array}{l}
\int_{\Omega}\left(-\mu^{-1} \nabla \phi \cdot \nabla \bar{\lambda}+\epsilon \omega^{2} \phi \bar{\lambda}\right) d A+\int_{\Gamma} \mu^{-1} \phi \partial_{n} \bar{\lambda} \partial s=0 \\
\quad \text { for all pseudoperiodic } \phi \text { and truncated strips } \Omega \\
\lambda \sim \sum_{m=m_{1}}^{m_{2}} \tau_{m} e^{i \kappa_{m} x} e^{i(m+\beta) y} \quad(x \rightarrow-\infty) \\
\lambda \sim \sum_{m=m_{1}}^{m_{2}} t_{m} e^{i \kappa_{m} x} e^{i(m+\beta) y}+\sum_{m=m_{1}}^{m_{2}} \rho_{m} e^{-i \kappa_{m} x} e^{i(m+\beta) y} \quad(x \rightarrow \infty)
\end{array}\right.
$$

This is an "antiradiating" scattering problem, in which the source field is a sum of plane waves traveling to the right, equal to the transmitted field in the scattering problem for $\psi$, and the "scattered field" is antiradiating. The problem can also be interpreted in a physical sense if we use the time harmonic factor $e^{i \omega t}$ instead of $e^{-i \omega t}$. Then the problem for $\lambda$ is just the scattering problem in which the transmitted field of $\psi$ is reversed and sent back toward the slab from the right. The left-hand side of (7) can now be rewritten and calculated using (9):

$$
\begin{aligned}
\Im\left[\int_{\Omega}\left(-\mu^{-1} \nabla \hat{\psi} \cdot \nabla \bar{\lambda}+\epsilon \omega^{2} \hat{\psi} \bar{\lambda}\right) d A+\right. & \left.\int_{\Gamma} \mu^{-1} \bar{\lambda} \partial_{n} \hat{\psi} d s\right]= \\
& =\Im \int_{\Gamma} \mu^{-1}\left(\bar{\lambda} \partial_{n} \hat{\psi}-\hat{\psi} \partial_{n} \bar{\lambda}\right) d s=\Im\left(4 \pi i \mu_{\mathrm{ext}}^{-1} \sum_{m=m_{1}}^{m_{2}} \kappa_{m} \bar{t}_{m} \hat{t}_{m}\right) .
\end{aligned}
$$




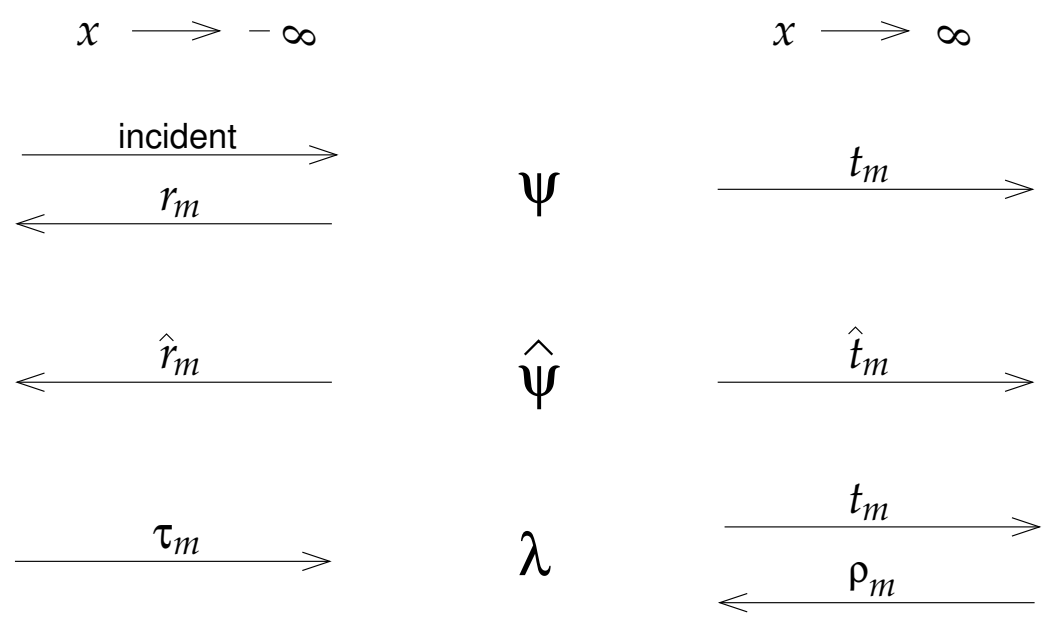

Figure 2. The far-field asymptotic behavior of the solution $\psi$ to the scattering problem (3), the perturbed field $\hat{\psi}$, and the solution $\lambda$ to the adjoint scattering problem (9).

On the other hand, we compute the right-hand side of (7) using the asymptotics in (3) and (5):

$$
\mu_{\mathrm{ext}}^{-1} \Im \int_{\Gamma_{+}}\left(\bar{\psi} \partial_{n} \hat{\psi}+\overline{\hat{\psi}} \partial_{n} \psi\right) d s=\mu_{\mathrm{ext}}^{-1} \Im \int_{\Gamma_{+}}\left(\bar{\psi} \partial_{n} \hat{\psi}-\hat{\psi} \partial_{n} \bar{\psi}\right) d s=\Im\left(4 \pi i \mu_{\mathrm{ext}}^{-1} \sum_{m=m_{1}}^{m_{2}} \kappa_{m} \bar{t}_{m} \hat{t}_{m}\right) .
$$

Thus, we have shown that if $\lambda$ satisfies problem (9), then (7) holds for all perturbations $\hat{\psi}$.

A word about the calculations of the integrals over $\Gamma$ and $\Gamma_{+}$that we have just performed: Each is a combination of integrals of the form

$$
\int_{0}^{2 \pi}\left(\bar{\phi}_{1} \partial_{x} \phi_{2}-\phi_{2} \partial_{x} \bar{\phi}_{1}\right) d y
$$

in which $\phi_{1}$ and $\phi_{2}$ are of the form

$$
\phi=\sum_{m=-\infty}^{\infty} c_{m} e^{\nu_{m} x} e^{i(m+\beta) y}
$$

where $\nu_{m}=i \kappa_{m}$ if the oscillatory modes propagate is to the right and $\nu_{m}=-i \kappa_{m}$ if they propagate to the left, and $\nu_{m}$ is real for the rest of the modes. Let $a_{m}$ be the coefficients for $\phi_{1}$ and $b_{m}$ the coefficients for $\phi_{2}$. Simple computation yields

$$
\int_{0}^{2 \pi}\left(\bar{\phi}_{1} \partial_{x} \phi_{2}-\phi_{2} \partial_{x} \bar{\phi}_{1}\right) d y= \begin{cases} \pm 4 \pi i \sum_{m=m_{1}}^{m_{2}} \kappa_{m} \bar{a}_{m} b_{m} & \text { if } \phi_{1} \text { and } \phi_{2} \text { propagate in the same direction, } \\ 0 & \text { if } \phi_{1} \text { and } \phi_{2} \text { propagate in opposite directions. }\end{cases}
$$

Using this rule and the diagram of asymptotics in Figure 2, the calculations above follow easily.

We now specialize the variational formula (8), which gives the first variation of $T$ as a function of $\epsilon$ and $\mu$, to specific classes of variations $\hat{\epsilon}$ and $\hat{\mu}$.

\subsection{Variation of the Values of $\epsilon$ and $\mu$}

Suppose that a period of the crystal slab consists of components $\left\{D_{j}\right\}_{j=1}^{n}$ with dielectric and magnetic constants $\epsilon_{j}$ and $\mu_{j}$ and exterior constants $\epsilon_{\text {ext }}$ and $\mu_{\text {ext }}$. Let $\hat{\epsilon}_{j}$ and $\widehat{\mu_{j}^{-1}}$ be perturbations of $\epsilon_{j}$ and $\mu_{j}^{-1}$, keeping the 
boundaries of the domains $D_{j}$ fixed. Then (8) gives

$$
\begin{aligned}
\hat{T} \approx \frac{\mu_{\mathrm{ext}}}{2 \pi \kappa_{\bar{m}}} \Im \sum_{j=1}^{n}\left[\widehat{\mu_{j}^{-1}} \int_{D_{j}} \nabla \psi \cdot \nabla \bar{\lambda} d A-\hat{\epsilon}_{j} \omega^{2} \int_{D_{j}} \psi \bar{\lambda} d A\right] \\
\quad=\frac{\mu_{\mathrm{ext}}}{2 \pi \kappa_{\bar{m}}} \Im \sum_{j=1}^{n}\left[\widehat{\mu_{j}^{-1}}\left(\mu_{j} \epsilon_{j} \omega^{2} \int_{D_{j}} \psi \bar{\lambda} d A-\int_{\partial D_{j}} \bar{\lambda} \partial_{n} \psi d s\right)-\hat{\epsilon}_{j} \omega^{2} \int_{D_{j}} \psi \bar{\lambda} d A\right]
\end{aligned}
$$

in which " $\approx$ " means equal to leading (linear) order in the variations $\hat{\epsilon}_{j}$ and $\widehat{\mu_{j}^{-1}}$. The last equality is a result of integration by parts.

\subsection{Variation of the Boundaries}

Now suppose that the constants $\epsilon_{j}$ and $\mu_{j}$ are fixed and that $v_{j}$ is a vector field on the boundary $\partial D_{j}$. Let $h$ be a small positive number, and let $T+\hat{T}$ be the transmission due to scattering by the perturbed crystal, in which the $j^{\text {th }}$ component has boundary defined by allowing $\partial D_{j}$ to flow along the vector field $v_{j}$ for a distance of $h$. Then (8) gives

$$
\hat{T}=\frac{\mu_{\mathrm{ext}}}{2 \pi \kappa_{\bar{m}}} \Im \sum_{j=1}^{n} \int_{\hat{D}_{j}}\left[ \pm\left(\mu_{j}^{-1}-\mu_{\mathrm{ext}}^{-1}\right) \nabla \psi \cdot \nabla \bar{\lambda} \mp\left(\epsilon_{j}-\epsilon_{\mathrm{ext}}\right) \omega^{2} \psi \bar{\lambda}\right] d A+o(h),
$$

where $\hat{D}_{j}$ is the region that passed from exterior to interior, in which case the upper sign is taken, or from interior to exterior, in which case the lower sign is taken. Taking the limit as $h \rightarrow 0$, we obtain the variational gradient of $T$ with respect to variations $v=\left\{v_{j}\right\}_{j=1}^{n}$ of the boundary $\partial D$ :

$$
\frac{\delta T}{\delta(\partial D)}=\lim _{h \rightarrow 0} \frac{\hat{T}}{h}=\frac{\mu_{\mathrm{ext}}}{2 \pi \kappa_{\bar{m}}} \Im \sum_{j=1}^{n} \int_{\partial D_{j}}\left[\left(\mu_{j}^{-1}-\mu_{\mathrm{ext}}^{-1}\right) \nabla \psi \cdot \nabla \bar{\lambda}-\left(\epsilon_{j}-\epsilon_{\mathrm{ext}}\right) \omega^{2} \psi \bar{\lambda}\right] v_{j} \cdot n d s
$$

\subsection{Variation of Circular Components}

We now specialize (12) further and take the $\partial D_{j}$ to be circles and compute the gradient of $T$ with respect to their radii $r_{j}$ and centers $\left(x_{j}, y_{j}\right)$. If we let $v_{j}=n$, then $v_{j} \cdot n=1$, and (12) gives

$$
\frac{\partial T}{\partial r_{j}}=\frac{\mu_{\text {ext }}}{2 \pi \kappa_{\bar{m}}} \Im \sum_{j=1}^{n} \int_{\partial D_{j}}\left[\left(\mu_{j}^{-1}-\mu_{\text {ext }}^{-1}\right) \nabla \psi \cdot \nabla \bar{\lambda}-\left(\epsilon_{j}-\epsilon_{\text {ext }}\right) \omega^{2} \psi \bar{\lambda}\right] d s .
$$

Letting $v_{j}=\vec{\imath}$, the unit normal vector pointing in the direction of the $x$-axis, and the $v_{j}=\vec{\jmath}$, the unit normal vector pointing in the direction of $y$-axis, we obtain

$$
\begin{gathered}
\frac{\partial T}{\partial x_{j}}=\frac{\mu_{\mathrm{ext}}}{2 \pi \kappa_{\bar{m}}} \Im \sum_{j=1}^{n} \oint_{\partial D_{j}}\left[\left(\mu_{j}^{-1}-\mu_{\mathrm{ext}}^{-1}\right) \nabla \psi \cdot \nabla \bar{\lambda}-\left(\epsilon_{j}-\epsilon_{\mathrm{ext}}\right) \omega^{2} \psi \bar{\lambda}\right] d y, \\
\frac{\partial T}{\partial y_{j}}=-\frac{\mu_{\mathrm{ext}}}{2 \pi \kappa_{\bar{m}}} \Im \sum_{j=1}^{n} \oint_{\partial D_{j}}\left[\left(\mu_{j}^{-1}-\mu_{\mathrm{ext}}^{-1}\right) \nabla \psi \cdot \nabla \bar{\lambda}-\left(\epsilon_{j}-\epsilon_{\mathrm{ext}}\right) \omega^{2} \psi \bar{\lambda}\right] d x,
\end{gathered}
$$

where the integration is taken counter-clockwise.

\section{NUMERICAL METHODS}

In this Section, we describe the algorithm and numerical methods by which we alter and optimize resonant behavior in a crystal slab and present an example. The basic algorithm goes as follows: 
1. Start with a structure whose transmission coefficient $T(\beta, \omega)$ exhibits resonant features to be optimized.

2. Choose a set of frequencies and directions in which the transmission or extrema in the transmission coefficient should move.

3. Find the variational gradient of the transmission with respect to the structural parameters.

4. Compute the smallest change in the parameters that gives the desired change in the transmission.

5. Increment the structure by the computed amount.

6. Update the set of frequencies if necessary.

7. Go back to 3 .

In our example, we begin with a square lattice of circular dielectric rods with $\epsilon=12$ and $\mu=1$ in air $(\epsilon=\mu=1)$, truncated to a slab three rods thick (Fig. 4), with a periodic channel running through it. The channel is created by adding an additional horizontal strip of air space between every three rows of rods. Thus, the new period in the $y$-direction is the "supercell" consisting of a $3 \times 3$ array of rods plus the additional horizontal strip.

Figure 3 shows the transmitted energy through the initial structure as a function of frequency, where the source field is a plane wave normally incident to the slab from the left. The region of low transmission from about $\omega=0.9$ to about $\omega=1.7$ coincides approximately with the bandgap for the initial square lattice. The periodic channel is the cause of the anomalous features - sharp spikes and the shallow hump, marked with an arrow, at the right end of the region. This hump corresponds to a mode going through the channel (see Ref. 3 for some discussion on such modes). We wish to modify the rod structure so as to sharpen this resonant scattering behavior, leaving the angle of incidence fixed. Figure 4 shows the modified structure and the resulting resonant spike in the transmission coefficient.

We use this example to explain our numerical methods for carrying out the algorithm.

1. To compute the transmission coefficient, we must solve the scattering problem (3), or equivalently (1), numerically. We do this using the method of boundary integrals based on Green's identities and the pseudo-periodic Green's function. Details can be found in Ref. 3.

2. We choose the frequency at which the peak of the transmission anomaly occurs, where we seek to increase the transmission, and two neighboring frequencies, where we seek to decrease the transmission.

3. We choose to restrict our admissible structures to arrays of circular rods with a fixed dielectric contrast of 12. Our design variables are the radii and centers of the rods. We compute the variational gradient by solving the scattering problems (3) and (9) numerically and computing the integrals (13), (14), and (15). We denote the gradient of the transmission with respect to these parameters by $\nabla_{\vec{p}} T$, where $\vec{p}$ is the 27 -dimensional vector of $(x, y)$-coordinates of the centers and the radii of the nine rods in the supercell.

4. Let $M$ denote the $3 \times 27$ matrix whose rows are equal to $\nabla_{\vec{p}} T$ at the three chosen frequencies, and let $\vec{b}$ denote the vector of desired increments in $T$ at these frequencies. We compute the smallest vector $\Delta \vec{p}$ that produces the change $\vec{b}$ by solving the $3 \times 3$ system

$$
M M^{t} \vec{x}=\vec{b}
$$

for $x$ and putting

$$
\Delta \vec{p}=M^{t} \vec{x} .
$$




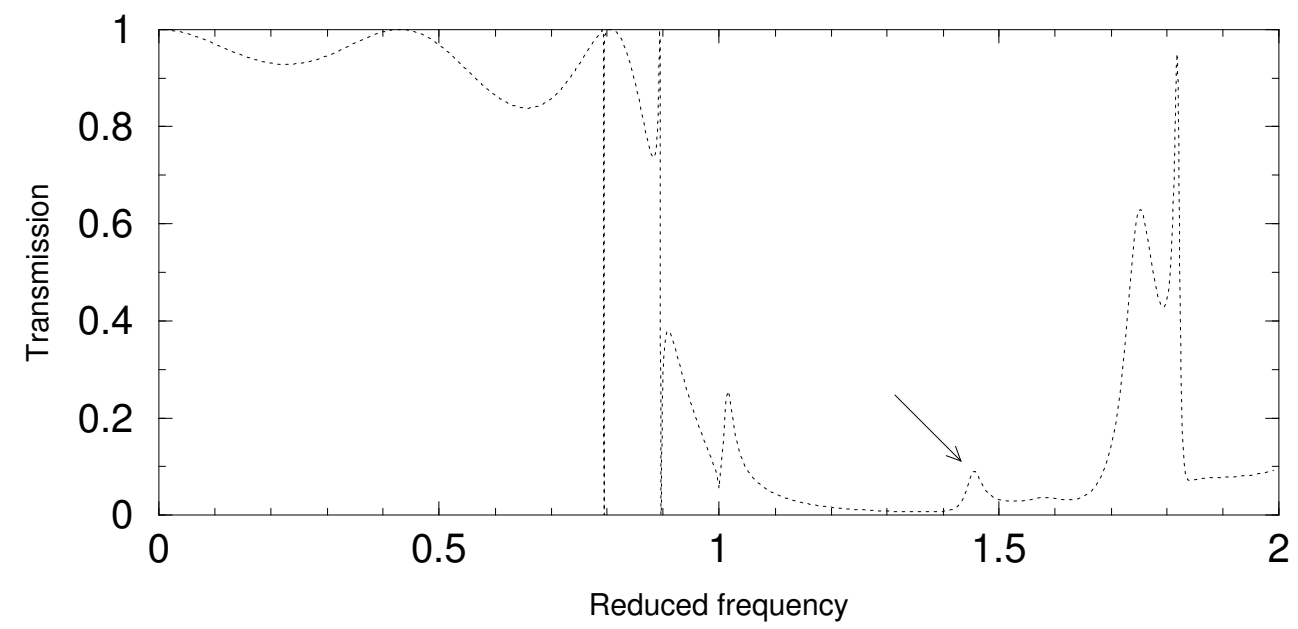

Figure 3. The percentage of energy $T$ of a plane wave source field that is transmitted through a slab is graphed as a function of the reduced frequency $\omega$. Two periods of the slab are shown in the first panel of Figure 4 . The periodic channel running through the slab is responsible for the anomalies in the transmission, including the hump indicated by the arrow, which we have sharpened by modifying the slab (Fig. 4).

6. As the structure is modified, the peak of the transmission anomaly may shift to a different frequency. We compute this shift as follows. Put $T_{1}=\partial T / \partial \omega$. The peak occurs when $T_{1}=0$, so we need to change $\omega$ by an amount $\Delta \omega$ that satisfies

$$
\frac{\partial T_{1}}{\partial \omega} \Delta \omega+\nabla_{\vec{p}} T_{1} \cdot \Delta \vec{p}=0
$$

or, solving for $\Delta \omega$,

$$
\Delta \omega=-\left(\frac{\partial T_{1}}{\partial \omega}\right)^{-1} \nabla_{\vec{p}} T_{1} \cdot \Delta \vec{p}
$$

\section{REFERENCES}

1. A. Krishnan, T. Thio, T. J. Kim, H. J. Lezec, T. W. Ebbesen, P. A. Wolf, J. Pendry, L. Martin-Moreno, and F. J. Garcia-Vidal, "Evanescently coupled resonance in surface plasmon enhanced transmission," Optics Communications 200, pp. 1-7, 2001.

2. S. Tikhodeev, A. Yablonskii, E. Muljarov, N. Gippius, and T. Ishihara, "Quasiguided modes and optical properties of photonic crystal slabs," Phys Rev B 66, pp. 045102:1-17, 2002.

3. M. Haider, S. Shipman, and S. Venakides, "Boundary-integral calculations of two-dimensional electromagnetic scattering in infinite photonic crystal slabs: Channel defects and resonances," SIAM J Appl Math 62-6, pp. 2129-2148, 2002.

4. S. Shipman and S. Venakides, "Resonance and bound states in photonic crystal slabs," SIAM J Appl Math in press, 2003.

5. S. Cox and D. Dobson, "Maximizing band gaps in two-dimensional photonic crystals," SIAM J Appl Math 59-6, pp. 2108-2120, 1999.

6. S. Cox and D. Dobson, "Band structure optimization of two-dimensional photonic crystals in h-polarization," J Comp Phys 158, pp. 214-224, 2000.

7. O. Pironneau, Optimal Shape Design for Elliptic Systems, Springer-Verlag, New York, 1984. 

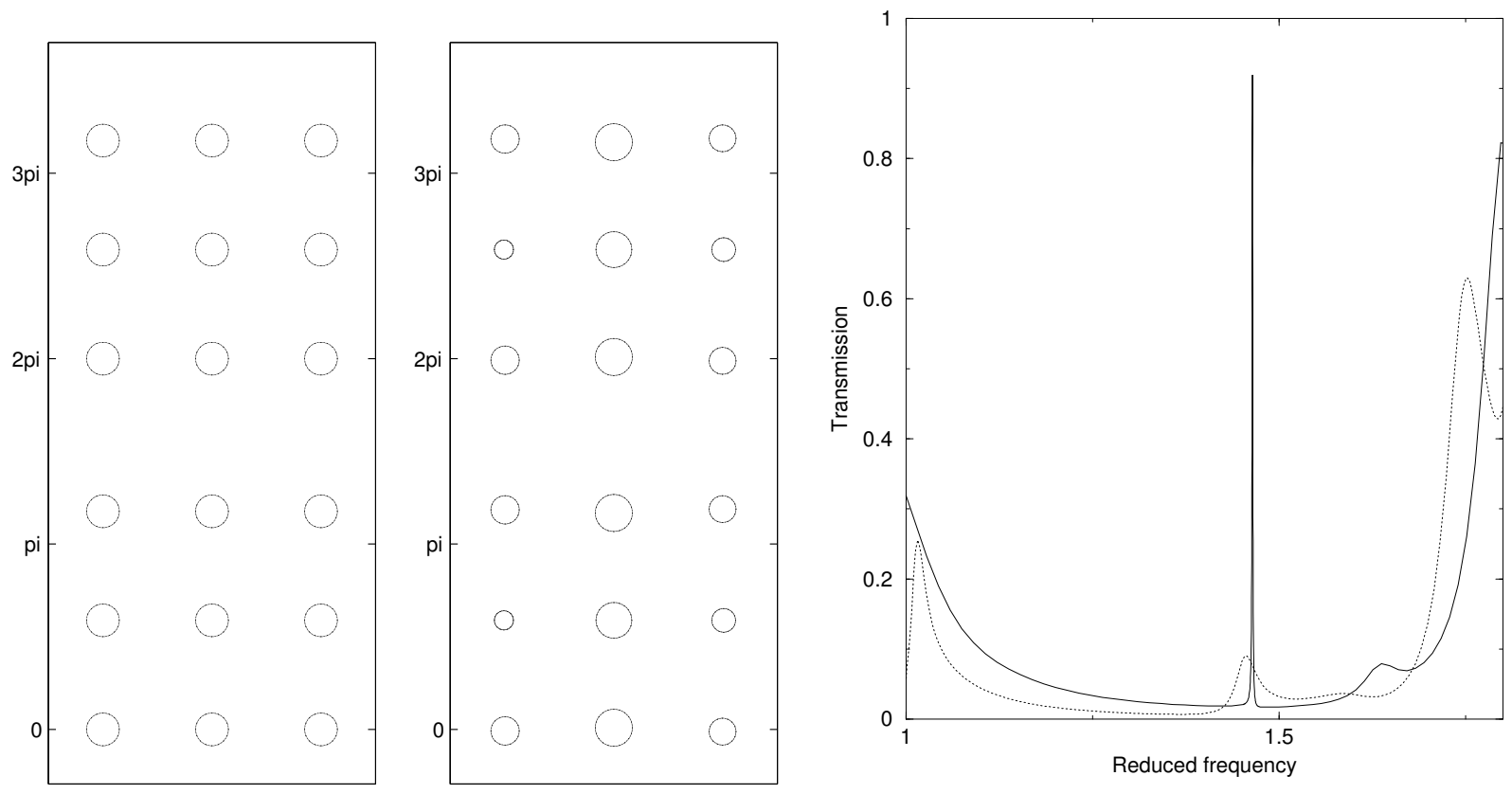

Figure 4. Two periods of the original photonic crystal slab (first panel) and the modified slab (second panel). The transmission coefficient of the original slab is shown as a dotted curve and that of the modified slab as a solid curve. The modification has transformed the shallow hump into a sharp resonance. 